\title{
Detection and Discrimination of Bioanalytes by Means of Colorimetric Sensor Array Based on Unmodified Gold and Silver Nanoparticles
}

\author{
Dongyang Li, Yanhua Dong, Bingyu Li and Yayan $\mathbf{W u}$ \\ Key Laboratory of Biomedical Information Engineering of Ministry of Education, School of Life Science and Technology, Xi'an Jiaotong University, 710049, Xi'an, PR \\ China
}

"Corresponding author: Yayan Wu, Key Laboratory of Biomedical Information Engineering of Ministry of Education, School of Life Science and Technology, Xi'an Jiaotong University, 710049, Xi'an, PR China, Tel: 7734316895; E-mail: 1991dongyangli@gmail.com

Received date: June 29, 2016; Accepted date: July 28, 2016; Published date: August 05, 2016

Copyright: (c) 2016 Li D, et al. This is an open-access article distributed under the terms of the Creative Commons Attribution License, which permits unrestricted use, distribution, and reproduction in any medium, provided the original author and source are credited.

\begin{abstract}
A new approach for sensing bioanalytes utilizing unmodified noble metal nanoparticles to fabricate a colorimetric sensor array is described. In this array, a series of gold and silver nanoparticles with various sizes whose color change based on the interactions of bioanalytes and nanoparticles, provide the unique response patterns for bioanalytes such as protein and bacteria which can be distinguished by naked eye. This work indicates that the colorimetric sensor array based on unmodified gold and silver nanoparticles has the potential for application in medical diagnostics.
\end{abstract}

Keywords: Nanoparticles; Bacteria; Bioanalytes

\section{Introduction}

The detection and identification of bioanalytes such as protein and bacteria are very important in the field of medical diagnostics. Enzyme-linked immunosorbent assays, PCR and mass spectrum are the most commonly used methods for bioanalytes sensing which are high costs, time consuming and impossible for multianalytes or mixtures. Optical chemical sensor array called "opto-chemical nose or tougue" is a promising sensing protocol and an alternative diagnostic platforms for a variety of bioanalytes. Rotello used conjugated fluorescent polymers array for protein detection [1]. Jiang reported a sensor array based on five fluorescent probes for the identification of bacteria [2]. Fluorescence sensor array is high sensitive; however, photo bleaching and excitation light source need are problematic Colorimetric sensor array is simple, fast, and effective way for the detection and discrimination of bioanalytes, providing optical response patterns of analytes readily discriminated by naked eye visualization. The special localized surface plasmon resonance (LSPR) effect makes noble metal nanoparticles an excellent choice of colorimetric sensor. The free electrons of gold nanoparticles (AuNPs) with different sizes and shapes can resonate with light of specific wavelength, so that they show different colors. When the condition of the solution is changed, the color will change too. Zhang developed a colorimetric sensor array for the detection of protein based on gold nanoparticles modified with aptamers [3]. But, all of the colorimetric methods required functionalized AuNPs. Quite a few studies have demonstrated that unmodified noble metal nanoparticles themselves can also interact with biological molecules such as proteins, non-specifically leading to a color change. We have developed a very simple colorimetric sensor array for the detection and discrimination of a range of bioanalytes using unmodified Nobel metal nanoparticles [4]. Here, this paper reviews the colorimetric sensor array and its application in bioanalytes sensing. Compared to previous colorimetric sensor array based on noble metal nanoparticles, this sensor array employs unmodified noble metal nanoparticles as sensing elements, which are much easier to be synthesized and cheaper. In addition, it has been demonstrated that a protein at various concentration can lead to different color changes. Therefore, this sensor array has the potential to be used for bioanalytes sensing.

\section{Colorimetric Sensor Array based on Unmodified Gold and Silver Nanoparticles}

Noble metal nanoparticles mainly include gold nanoparticles (AuNPs) and silver nanoparticles (AgNPs), having the most outstanding property is LSPR. Typically, the aggregation of AuNPs and the changes in color of AuNPs occur when the analyte interacts with AuNPs. This LSPR property has been implemented to build colorimetric biosensor and realize visualization detection for various bioanalytes. It has been reported that nanoparticles with different surface properties (e.g. size, shape, surface charge, and coating material) have different interactions with proteins $[5,6]$. Nanoparticles with different sizes have different surface areas and curvature which have effect on protein folding, because various surface-tovolume ratios have different non-specific interaction of amino acid residue of protein and nanoparticels surface. Therefore, we synthesized five unmodified AuNPs and two unmodified AgNPs with various sizes according to reported literatures [6] to investigate the interactions between AuNPs and bioanalytes. To synthesize AuNPs, chloroauric acid was reduced by sodium citrate. While to synthesize the AgNPs, Tollens' reagent was reduced by glucose. The size of nanoparticles can be easily changed by adjusting the ratio of oxidant and reductant. Their diameters of AuNPs 1, AuNPs 2, AuNPs 3, AuNPs 4, AuNPs 5, AgNPs 1 and AgNPs 2 are $15 \mathrm{~nm}, 25 \mathrm{~nm}, 35 \mathrm{~nm}, 50 \mathrm{~nm}, 60 \mathrm{~nm}, 25 \mathrm{~nm}$ and 31 nm respectively. In fact, what is called "unmodified" AuNPs are citratestabilized AuNPs because they are not functionalized AuNPs or AgNPs. Five unmodified AuNPs with different sizes (NP1: $15 \mathrm{~nm}$; NP2:25 nm; NP3:35 nm; NP4:50 nm; NP5: $60 \mathrm{~nm}$ ) are chosen to study the interactions of AuNPs and proteins. When proteins including cytochrome C (Cyt C), myoglobin (Mb), trypsin (Try), pepsin (Pep), lysozyme (Lys), papain (Pap), Casein (Cas), bovine serum albumin (BSA), lipase (Lip), and hemoglobin (Hb), incubated with AuNPs, 
respectively, the aggregation of AuNPs led to the color changes from red to purple, which can be visualized by the naked eye. As shown in Figure 1, the color change profiles are unique fingerprints for each specific protein at given concentration. Moreover, various concentrations of proteins also result in different color patterns. Therefore, a colorimetric sensor array was designed based on five unmodified AuNPs and two unmodified AgNPs for the detection and discrimination of bioanalytes. These noble metal nanoparticles were used as the sensing elements, which served not only as the receptors but also the indicators. They could bind diversely in the presence of proteins and distinct absorbance response patterns for the proteins were created, as shown in Figure 2.

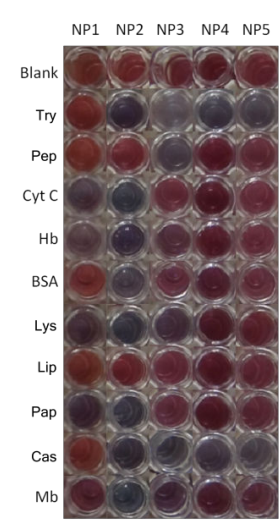

(a) $0.5 \mu \mathrm{M}$

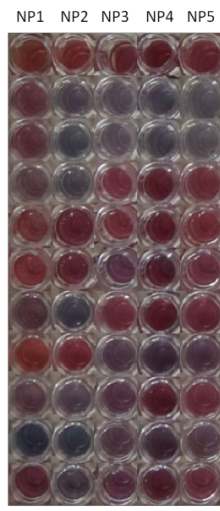

(b) $5 \mu \mathrm{M}$

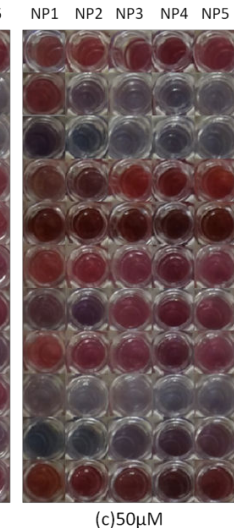

(c) $50 \mu \mathrm{M}$
Figure 1: The color change patterns for five unmodified AuNPs of various sizes in the presence of different kinds and concentrations of proteins [4].

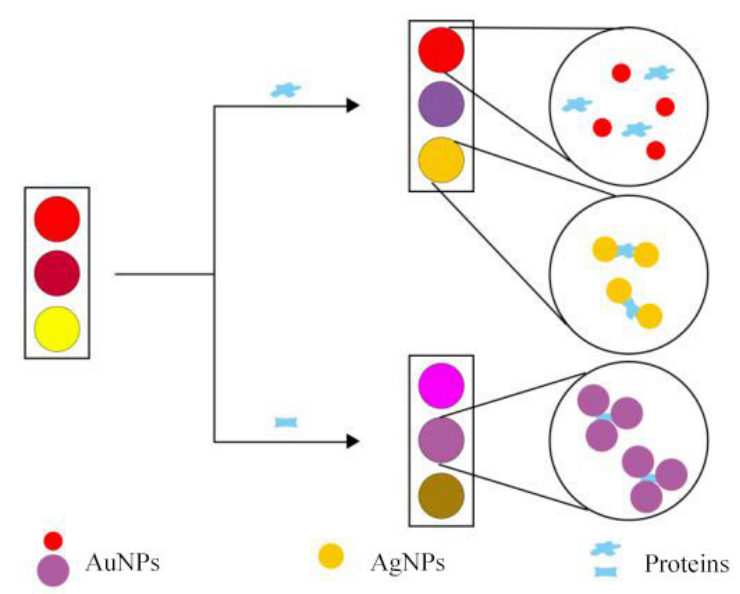

Figure 2: Schematic diagrams of the interaction between protein and unmodified Nobel metal nanoparticles.

\section{Discrimination of Bioanalytes}

In order to evaluate the discrimination ability of the proposed sensor array, ten proteins at concentration of $0.5 \mu \mathrm{M}$ were identified by absorbance. The proteins include Cyt C, Mb, Try, Pep, Lys, Pap, Cas, $\mathrm{BSA}$, Lip and $\mathrm{Hb}$, which have different molecular weights, isoelectric points (pIs) and oligomeric states. The results show that different proteins have their unique absorbance response patterns due to the interactions of the nanoparticles with proteins. The raw data obtained from the optical response patterns were subjected to linear discriminant analysis (LDA). LDA is a statistic method to recognize the linear combination of features that can differentiate different classes of objects. Herein, LDA was employed to reduce the size of the training matrix (7 sensing elements $\times 10$ proteins $\times 3$ replicates). Three canonical factors were obtained and visualized as a three-dimensional plot. According to this plot, all the proteins were distinguished with accuracy of $100 \%$, as illustrated in Figure $3 a$.

After successful discrimination of proteins by the proposed sensor array, the next challenge was to sense the real biosamples. As we know, there are a lot of proteins on the surface of bacteria and human cancer cells. Therefore, it is assumed that this sensor array could be used for the identification of bacteria and human cancer cells. To test this assumption, seven bacteria and four human cancer cells were used as real samples to be detected by the proposed sensor array. As shown in Figure $3 b$ and $3 c$, bacteria and human cells could be differentiated with accuracy of $100 \%$. This study indicates the potential of the sensor array to be used for diagnose of cancers and detection of bacteria.

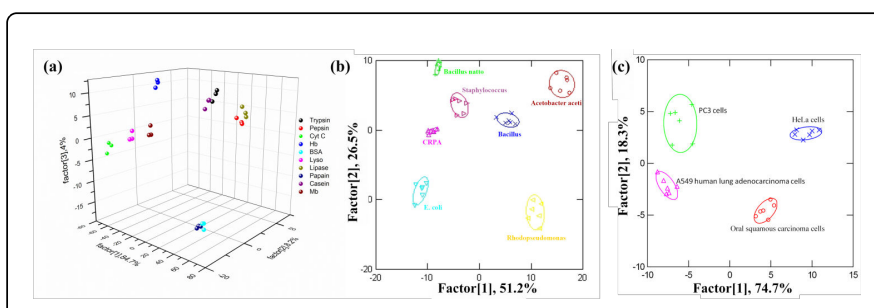

Figure 3: Identification of bioanalytes [4]. (a) Canonical score plot for three factors of simplified absorbance response patterns obtained with the noble metal nanoparticle-based array against 0.5 $\mu \mathrm{M}$ proteins: Cyt C, Mb, Try, Pep, Lys, Pap, Cas, BSA, Lip and Hb. All proteins were clearly distinguished. (b) Canonical score plot for three factors of simplified absorbance response patterns against bacterias: E. coli, CRPA, Acetobacter aceti, Rhodopseudomonas, Bacillus natto, Staphylococcus, and Bacillus (0.05 OD in $200 \mu \mathrm{L}$ ). All the bacterias were distinguished perfectly. (c) Canonical score plot for three factors of simplified absorbance response patterns against human cancer cells: Oral squamous carcinoma cells, HeLa cells, PC3 cells, and A549 human lung adenocarcinoma cells (5000 cells in $200 \mu \mathrm{L}$ ).

\section{Conclusion}

Based on the LSPR of noble metal nanoparticles, a colorimetric sensor array was constructed for the identification of proteins. The sensor array is composed of seven sensing elements, including five unmodified AuNPs and two unmodified AgNPs with various sizes. Ten proteins were successfully distinguished by the proposed colorimetric sensor array. The presence of proteins induced obvious color change of AuNPs, which could be easily recognized by naked eyes. Two real samples including seven bacteria and four cancer cells were also employed to test the discrimination ability of this sensor array. This sensor array is more simple and cheaper that other noble metal nanoparticles-based sensor arrays. Besides, the size of AuNPs and AgNPs can be easily adjusted, which makes it easier to select as many sensor elements as possible. One of the limitations of this sensor array 
Citation: Li D, Dong Y, Li B, Wu Y (2016) Detection and Discrimination of Bioanalytes by Means of Colorimetric Sensor Array Based on Unmodified Gold and Silver Nanoparticles. J Bacteriol Parasitol 7: 283. doi:10.4172/2155-9597.1000283

Page 3 of 3

is that the sensing elements (AuNPs and AgNPs) can' not be used repeatedly. This is also a common limitation of most sensor arrays. Even though there are some limitations, this research indicates the potential of unmodified noble metal nanoparticles for the detection and discrimination of proteins and bacteria.

\section{References}

1. Avinash B, Oscar RM, Ronnie P, Ik-Bum K, Djosepy J, et al. (2009) Arraybased sensing of normal, cancerous and metastatic cells using conjugated fluorescent polymers. Journal of the American Chemical Society 132 : 1018-1022.

2. Wenwen C, Qizhai L, Wenshu Z, Fang H, Guanxin Z, et al. (2014) Identification of bacteria in water by a fluorescent array. Angewandte Chemie 126 : 13954-13959.
3. Yuexiang L, Yueying L, Suge Z, Song W, Sichun Z, et al. (2013) Aptamerbased plasmonic sensor array for discrimination of proteins and cells with the naked eye. Anal Chem 85 : 6571-6574.

4. Dongyang L, Yanhua D, Bingyu L, Yayan W, Kai W, et al. (2015) Colorimetric sensor array with unmodified noble metal nanoparticles for naked-eye detection of proteins and bacteria. Analyst 140 : 7672-7677.

5. AubinTam ME, Hamad-Schifferli K (2008) Structure and function of nanoparticle-protein conjugates. Biomed Mater 3: 034001.

6. Daniel MC, Astruc D (2004) Gold nanoparticles: assembly, supramolecular chemistry, quantum-size-related properties, and applications toward biology, catalysis, and nanotechnology. Chem Rev 104: 293-346. 